\title{
Some Constructions of General Covering Designs
}

\author{
Federico Montecalvo \\ School of Mathematical Sciences \\ Queen Mary, University of London \\ London E1 4NS, U.K. \\ F.Montecalvo@qmul.ac.uk
}

Submitted: Jun 10, 2011; Accepted: Aug 23, 2012; Published: Sep 6, 2012

Mathematics Subject Classification: 05B40

\begin{abstract}
Given five positive integers $v, m, k, \lambda$ and $t$ where $v \geqslant k \geqslant t$ and $v \geqslant m \geqslant t$, a $t$ - $(v, k, m, \lambda)$ general covering design is a pair $(X, \mathcal{B})$ where $X$ is a set of $v$ elements (called points) and $\mathcal{B}$ a multiset of $k$-subsets of $X$ (called blocks) such that every $m$-subset of $X$ intersects (is covered by) at least $\lambda$ members of $\mathcal{B}$ in at least $t$ points.

In this article we present new constructions for general covering designs and we generalize some others. By means of these constructions we will be able to obtain some new upper bounds on the minimum size of such designs.
\end{abstract}

Keywords: covering design; Turán system; lotto design; block design

\section{Introduction}

Given five positive integers $v, m, k, \lambda$ and $t$ where $v \geqslant k \geqslant t$ and $v \geqslant m \geqslant t$, a $t$ - $(v, k, m, \lambda)$ general covering design (or general cover) is a pair $(X, \mathcal{B})$ where $X$ is a set of $v$ elements (called points) and $\mathcal{B}$ a multiset of $k$-subsets of $X$ (called blocks) such that every $m$-subset of $X$ intersects (is covered by) at least $\lambda$ members of $\mathcal{B}$ in at least $t$ points.

It is easy to verify that a $t-(v, k, m, \lambda)$ general cover is also a $(t-1)-(v, k, m-1, \lambda)$ general cover. A $t$ - $(v, k, m, \lambda)$ general covering design $(X, \mathcal{B})$ is said to be optimal if:

$$
|\mathcal{B}|=\min \{|\mathcal{A}|: \text { there is a } t \text { - }(v, k, m, \lambda) \text { general covering design }(X, \mathcal{A})\} .
$$

In this case, the cardinality of $\mathcal{B}$ is called the general covering number and denoted by $C_{\lambda}(v, k, t, m)$.

Given a $t$ - $(v, k, m, 1)$ general covering design $(X, \mathcal{B})$, the set $\mathcal{C}=\{X \backslash B: B \in \mathcal{B}\}$ is said to be the collection of the coblocks of $(X, \mathcal{B})$ and the pair $(X, \mathcal{C})$ is called the complement of $(X, \mathcal{B})$. 
Applications to error-trapping decoding, data compression and lottery systems have led many special cases of general covering designs to be investigated. Let us describe the most studied in the literature:

Covering Designs: When $m=t$ and $\lambda=1$, a $t$ - $(v, k, m, \lambda)$ general covering design is said to be a $(v, k, t)$ covering design. The general covering number is simply called covering number and denoted by $C(v, k, t)$. There is an extensive literature on covering designs. For an excellent survey please refer to [19, 24, 25]. Covering designs are applied to error-trapping decoding [10]. Here the number of the blocks determines the complexity of the decoding procedure. So, optimal covering designs are of special interest.

Turán Systems: When $k=t$ and $\lambda=1$, a $t$ - $(v, k, m, \lambda)$ general covering design is said to be a $(v, k, m)$ Turán system. The general covering number is called Turán number and denoted by $T(v, k, m)$. By taking the coblocks of a $(v, k, t)$ covering design, we always obtain a $(v, v-k, v-t)$ Turán system. Conversely, if we take the coblocks of a $(v, k, m)$ Turán system we always obtain a $(v, v-k, v-m)$ covering design. Therefore: $T(v, k, m)=C(v, v-k, v-m)$ and $C(v, k, t)=T(v, v-k, v-t)$. For a survey please refer to $[8,13,28]$.

Lotto Designs: When $\lambda=1$, a $t$ - $(v, k, m, \lambda)$ general covering design is said to be a $(v, k, t, m)$ lotto design (or cover). We will generally use the latter definition in the following sections. The general covering number is called lotto (or cover) number and denoted either by $L(v, k, t, m)$ or by $C(v, k, t, m)$. From the definition, both covering designs and Turán systems can be seen as special cases of lotto designs where $m=t$ and $k=t$ respectively. Therefore $C(v, k, t)=C(v, k, t, t)=T(v, v-$ $k, v-t)$ and $T(v, k, m)=C(v, k, k, m)=C(v, v-k, v-m)$. As the name suggests, lotto designs find application to national lotteries $[6,11,17]$, but they are also applied to data compression algorithms, as described in [15]. Several studies have focused on establishing upper and lower bounds on $C(v, k, t, m)$. Currently, the situation is as follows:

- Only for few values of $v, k, t$ and $m$ the cover number $C(v, k, t, m)$ has been found (see $[3,7,8,22]$ ).

- Constructions and lotto tables have been published in international journals (see $[4,6,11,22])$.

- Upper bounds on $C(v, k, t, m)$ are available on web sites (see $[5,16,18])$.

- Results on lower bounds have also been published (see [17, 23]).

General covers should not be confused with a class of objects called generalized covering designs which were recently introduced by Bailey et al. in [1]. Generalized covering designs simultaneously generalize covering designs and covering arrays. For further information and details on this class of objects, the reader is referred to the aforementioned reference. 


\section{Background}

In this section we present definitions and known results on design theory which will be used throughout this article.

Definition 1. A block design is a pair $(X, \mathcal{B})$ such that:

1. $X$ is a set of elements called points.

2. $\mathcal{B}$ is a multiset (collection) of non-empty subsets of $X$ called blocks.

The cardinality of $X$ is said to be the order of a block design $(X, \mathcal{B})$. Two block designs $(X, \mathcal{A})$ and $(X, \mathcal{B})$ are called disjoint if $\mathcal{A} \cap \mathcal{B}=\emptyset$. The product of two block designs $\left(X_{1}, \mathcal{A}\right)$ and $\left(X_{2}, \mathcal{B}\right)$ is defined as $\left(X_{1} \cup X_{2}, \mathcal{A B}\right)$ where $\mathcal{A B}=\{A \cup B: A \in \mathcal{A}, B \in \mathcal{B}\}$.

Definition 2. A $t$ - $(v, k, \lambda)$-design is a a pair $(X, \mathcal{B})$ where $X$ is a set of $v$ elements (called points) and $\mathcal{B}$ a multiset of $k$-subsets of $X$ (called blocks) such that every $t$-subset of $X$ is contained in exactly $\lambda$ blocks.

The general term $t$-design is often used to indicate any $t$ - $(v, k, \lambda)$-design. When $\lambda=1$, a $t$ - $(v, k, 1)$-design is often called a Steiner system and denoted by $S(v, k, t)$. If $t=2$ and $k=3$, a Steiner system is called a Steiner triple system and denoted by $\operatorname{STS}(v)$ and if $t=3$ and $k=4$ it is called a Steiner quadruple system and denoted by $\operatorname{SQS}(v)$.

When $\lambda>1$, the union of two collections of blocks $\mathcal{A}$ and $\mathcal{B}$ of $t$-designs (or general covering designs) is a multiset union. Therefore, if a block $C$ appears $r_{1}$ times in $\mathcal{A}$ and $r_{2}$ times in $\mathcal{B}, C$ will appear $\max \left\{r_{1}, r_{2}\right\}$ times in $\mathcal{A} \cup \mathcal{B}$.

Many results on the necessary and sufficient conditions for the existence of $t$-designs have been found. Here we report the one on Steiner triple systems:

Theorem 3. [20] There exists an $\operatorname{STS}(v)$ if and only if $v \equiv 1,3(\bmod 6), v \geqslant 7$.

A $t$-design $(X, \mathcal{B})$ is said to be $\alpha$-resolvable if there exists a partition of the collection $\mathcal{B}$ into parts called $\alpha$-parallel classes (or $\alpha$-resolution classes) such that each point of $X$ occurs exactly in $\alpha$ blocks in each class. When $\alpha=1, \alpha$ is omitted.

Another interesting concept is the one of $i$-resolvable designs:

Definition 4. A Steiner system $S(v, k, t)$ is called $i$-resolvable, $0<i<t$, if the collection of its blocks can be partitioned into Steiner systems $S(v, k, i)$.

With regard to $i$-resolvable designs, the following two important theorems hold:

Theorem 5. [2] For any positive integer $n$ there exists a 2-resolvable SQS $\left(4^{n}\right)$.

Theorem 6. [30] For any positive integer $n$ there exists a 2-resolvable $\mathrm{SQS}\left(2 p^{n}+2\right)$, $p \in\{7,31,127\}$.

When $k=2$, we often talk in terms of graphs rather than designs. 
Definition 7. The complete graph of order $n$, denoted by $K_{n}$, is a regular graph with $n$ vertices such that each pair of vertices is an edge.

The number of edges of the complete graph $K_{n}$ is $\frac{n(n-1)}{2}$, that is, all the possible pairs of vertices.

A 1-factor of a graph $G$ is a set $E$ of edges such that every vertex of $G$ is incident to exactly one edge of $E$. A 1-factorization is a partition of the edges of a graph into 1-factors. In term of designs, a 1-factorization of the complete graph $K_{n}$ corresponds to a partition of the Steiner system $S(n, 2,2)$ (i.e. the set of all the pairs from $n$ ) into parallel classes. Clearly, $n$ must be even.

A definition of resolvability can be extended to covering designs as follows:

Definition 8. A $(v, k, t)$ covering design $(X, \mathcal{B})$ is resolvable if $\mathcal{B}$ can be partitioned into parts called parallel classes (or resolution classes) each of which in turn partitions $X$.

The number of blocks in a parallel class is necessarily $v / k$.

Let $r(q, k)$ denote the minimum number of parallel classes in a resolvable $(k q, k, 2)$ covering design. When $q=1, r(q, k)$ is trivially equal to 1 . The following results hold:

Theorem 9. [32] When $q>1, r(q, k) \geqslant q+1$. Equality holds if and only if $q$ divides $k$ and $q$ is the order of an affine plane.

For small values of $q$ :

Theorem 10. [32]

1. $r(2, k)=3$ if $k$ is even, 4 if $k$ is odd;

2. $r(3, k)=4$ if $k \equiv 0(\bmod 3), 5$ otherwise;

3. $r(4, k)=5$ if $k \equiv 0(\bmod 4), 7$ if $k \in\{2,3\}, 6$ otherwise.

Another interesting concept is the one of large set of coverings. Given a set $X$ of size $v$ and a positive integer $k$, let $\left(\begin{array}{l}X \\ k\end{array}\right)$ be the set of all $k$-subsets of $X$ and let $\mu(v, k)$ denote the minimum number of optimal $(v, k, k-1)$ covering designs $\left(X, \mathcal{B}_{1}\right),\left(X, \mathcal{B}_{2}\right), \ldots,\left(X, \mathcal{B}_{\mu(v, k)}\right)$ such that $\bigcup_{i=1}^{\mu(v, k)} \mathcal{B}_{i}=\left(\begin{array}{l}X \\ k\end{array}\right)$. Let $\lambda(v, k)$ denote instead the maximum number of disjoint optimal $(v, k, k-1)$ covering designs defined on $X$. Then a large set of coverings is obtain when $\lambda(v, k)=\mu(v, k)$. The following results hold:

Theorem 11. [14]

1. $\mu(8,4)=6$.

2. $\mu(9,4)=6$.

3. $\mu(10,4) \leqslant 10$.

In the following sections, given a partition $X_{1}, \ldots, X_{n}$ of a set $X$ of size $v$, a positive integer $m \leqslant v$, and $n$ positive integers $a_{1} \leqslant\left|X_{1}\right|, \ldots, a_{n} \leqslant\left|X_{n}\right|$ such that $\sum_{i=1}^{n} a_{i}=m$, we will assume that $\left[a_{1}, \ldots, a_{n}\right]$ denotes the subset of $\left(\begin{array}{l}X \\ m\end{array}\right)$ whose elements $M$ satisfy $\left|M \cap X_{i}\right|=a_{i}$, for $1 \leqslant i \leqslant n$. 


\section{Point Splicing Constructions}

Etzion et al. [15] described a construction for constant weight covering codes called onebit splicing. It was actually a construction for $(v, k, m)$ Turán systems. The objective was to start from a Turán system of order $v$ to obtain a Turán system of order $v+1$. In the next section we present a simple generalization: We start from a general covering design of order $v$ to obtain a general covering design of order $v+n$.

\subsection{Point Splicing Construction for $t-(v, k, m, \lambda)$ General Covers}

Let $(X, \mathcal{B})$ be a $t$ - $(v, k, m, \lambda)$ general covering design and $n$ be the size of a set $S$ such that $X \cap S=\emptyset$ and $n \leqslant k-t+1$. For every $x \in X$, define $\mathcal{B}(x)=\{B \backslash\{x\}: B \in \mathcal{B}, x \in B\}$. Choose $a \in X$ such that for any $x \in X$ we have $|\mathcal{B}(a)| \leqslant|\mathcal{B}(x)|$. Let $(X \backslash\{a\}, \mathcal{C})$ be a $(t-2)-(v-1, k-n-1, m-2, \lambda)$ general covering design and $\mathcal{B}_{1}, \mathcal{B}_{2}$, and $\mathcal{B}_{3}$ be three collections of blocks as defined below:

$$
\begin{aligned}
& \mathcal{B}_{1}=\mathcal{B} . \\
& \mathcal{B}_{2}=\{B \cup\{s\}: B \in \mathcal{B}(a), s \in S\} . \\
& \mathcal{B}_{3}=\{C \cup S \cup\{a\}: C \in \mathcal{C}\} .
\end{aligned}
$$

Our objective is to obtain a $t$ - $(v+n, k, m, \lambda)$ general covering design on the set $(X \cup S)$ and we claim that $\left(X \cup S, \mathcal{B}_{1} \cup \mathcal{B}_{2} \cup \mathcal{B}_{3}\right)$ meets the objective.

Theorem 12. $\left(X \cup S, \mathcal{B}_{1} \cup \mathcal{B}_{2} \cup \mathcal{B}_{3}\right)$ is a $t-(v+n, k, m, \lambda)$ general covering design.

Proof. Let $M$ be an $m$-subset of $X \cup S$ :

If $M \cap(S \cup\{a\})=\emptyset$ or $\{a\}$, then there exist at least $\lambda$ blocks in $\mathcal{B}_{1}$ that cover $M$ in $t$ points.

If $M \cap(S \cup\{a\})=\{s\}, s \in S$, then there exist at least $\lambda$ blocks in $\mathcal{B}_{1} \cup \mathcal{B}_{2}$ that cover $M$ in $t$ points.

If $|M \cap(S \cup\{a\})|=\delta, 2 \leqslant \delta<t$, then there exist at least $\lambda$ blocks in $\mathcal{B}_{3}$ that cover $M$ in $t$ points because $(X \backslash\{a\}, \mathcal{C})$ is a $(t-2)-(v-1, k-n-1, m-2, \lambda)$ general covering design and therefore a $(t-\delta)-(v-1, k-n-1, m-\delta, \lambda)$ general covering design as well.

If $|M \cap(S \cup\{a\})|=\delta \geqslant t$, then $M$ is clearly covered by each block $B \in \mathcal{B}_{3}$.

Therefore $\left(X \cup S, \mathcal{B}_{1} \cup \mathcal{B}_{2} \cup \mathcal{B}_{3}\right)$ is a $t$ - $(v+n, k, m, \lambda)$ general covering design.

By counting arguments, $\min _{x \in X}|\mathcal{B}(x)| \leqslant\left\lfloor\frac{k|\mathcal{B}|}{v}\right\rfloor$, therefore, as a consequence of the construction above:

$C_{\lambda}(v+n, k, t, m) \leqslant n\left\lfloor\frac{k}{v} C_{\lambda}(v, k, t, m)\right\rfloor+C_{\lambda}(v, k, t, m)+C_{\lambda}(v-1, k-n-1, t-2, m-2)$. 


\subsection{Point Splicing Construction for $(v, 6,4,6)$ Covers}

We introduce a point splicing construction specific for $(v, 6,4,6)$ covers. Similar in spirit to a construction for $(v, 4,6)$ Turán systems presented by Etzion et al. [15], it permits us to obtain a $(v+3,6,4,6)$ cover from a $(v, 6,4,6)$ cover.

Let $(X, \mathcal{B})$ be a $(v, 6,4,6)$ cover. For every $x \in X$, let $\mathcal{B}(x)$ be defined as in Section 3.1. Choose $a \in X$ such that for any $x \in X$ we have $|\mathcal{B}(a)| \leqslant|\mathcal{B}(x)|$. Let $\{b, c, d\}$ be a set such that $X \cap\{b, c, d\}=\emptyset$. Let $X_{1,1}, X_{1,2}, X_{2,1}, X_{2,2}, X_{3,1}, X_{3,2}$ be a partition of $X \backslash\{a\}$. Then take the following block designs where a pair-by-triple covering denotes a covering design with $k=3$ and $t=2$ and a pair-by-quadruple covering one with $k=4$ and $t=2$ :

Three pair-by-triple coverings $\left(X_{1,1} \cup X_{2,1}, \mathcal{C}_{1}\right),\left(X_{1,2} \cup X_{2,2}, \mathcal{C}_{2}\right)$ and $\left(X_{3,1} \cup X_{3,2}, \mathcal{C}_{3}\right)$.

Three pair-by-triple coverings $\left(X_{1,1} \cup X_{2,2}, \mathcal{D}_{1}\right),\left(X_{1,2} \cup X_{2,1}, \mathcal{D}_{2}\right)$ and $\left(X_{3,1} \cup X_{3,2}, \mathcal{D}_{3}\right)$.

Three pair-by-quadruple coverings $\left(X_{1,1} \cup X_{1,2}, \mathcal{E}_{1}\right),\left(X_{2,1} \cup X_{2,2}, \mathcal{E}_{2}\right),\left(X_{3,1} \cup X_{3,2}, \mathcal{E}_{3}\right)$.

The designs above have the following properties:

1. $\left(X \backslash\{a\}, \bigcup_{i=1}^{3} \mathcal{C}_{i}\right)$ is a $(v-1,3,2,4)$ cover.

2. $\left(X \backslash\{a\}, \bigcup_{i=1}^{3} \mathcal{D}_{i}\right)$ is a $(v-1,3,2,4)$ cover.

3. $\left(X \backslash\{a\}, \bigcup_{i=1}^{3} \mathcal{E}_{i}\right)$ is a $(v-1,4,2,4)$ cover.

4. For any triple $\{x, y, z\} \subset X \backslash\{a\}$, there exists a block $B$ in $\bigcup_{i=1}^{3} \mathcal{C}_{i} \cup \mathcal{D}_{i} \cup \mathcal{E}_{i}$ such that $|B \cap\{x, y, z\}| \geqslant 2$.

We can now proceed to build a $(v+3,6,4,6)$ cover. Define:

$$
\begin{aligned}
& \mathcal{B}_{1}=\mathcal{B} . \\
& \mathcal{B}_{2}=\{B \cup\{p\}: B \in \mathcal{B}(a), p \in\{b, c, d\}\} . \\
& \mathcal{B}_{3}=\left\{C \cup\{a, b, c\}: C \in \bigcup_{i=1}^{3} \mathcal{C}_{i}\right\} . \\
& \mathcal{B}_{4}=\left\{D \cup\{a, b, d\}: D \in \bigcup_{i=1}^{3} \mathcal{D}_{i}\right\} . \\
& \mathcal{B}_{5}=\left\{E \cup\{c, d\}: E \in \bigcup_{i=1}^{3} \mathcal{E}_{i}\right\} .
\end{aligned}
$$

Theorem 13. $\left(X \cup\{b, c, d\}, \bigcup_{i=1}^{5} \mathcal{B}_{i}\right)$ is a $(v+3,6,4,6)$ cover.

Proof. Let $M$ be a 6-subset of $X \cup\{b, c, d\}$ :

If $M \cap\{a, b, c, d\}=\emptyset$ or $\{a\}$ then $M$ is covered by some block $B \in \mathcal{B}_{1}$.

If $M \cap\{a, b, c, d\}=\{b\}$ or $\{c\}$ or $\{d\}$, then $M$ is covered by some block $B \in \mathcal{B}_{1} \cup \mathcal{B}_{2}$.

If $|M \cap\{a, b, c, d\}|=2$, then there exists $P \in\{\{a, b, c\},\{a, b, d\},\{c, d\}\}$ such that $P \supseteq(M \cap\{a, b, c, d\})$. Let $P=\{a, b, c\}$ (the cases when $P=\{a, b, d\}$ or $P=\{c, d\}$ are similar). From property $1, M$ is covered by some block $B \in \mathcal{B}_{3}$. 
If $M \cap\{a, b, c, d\}=\{a, b, c\}$, then $M$ is covered by some block $B \in \mathcal{B}_{3}$.

If $M \cap\{a, b, c, d\}=\{a, b, d\}$, then $M$ is covered by some block $B \in \mathcal{B}_{4}$.

If $M \cap\{a, b, c, d\}=\{a, c, d\}$, then $M$ is a set of the form $\{a, c, d, x, y, z\}$ where $\{x, y, z\}$ is any triple of $X \backslash\{a\}$. From property 4, it follows that there exists a block $T \in \bigcup_{i=1}^{3} \mathcal{C}_{i} \cup \mathcal{D}_{i} \cup \mathcal{E}_{i}$ such that $|T \cap\{x, y, z\}| \geqslant 2$. Moreover, since $\{a, c, d\}$ pairwise intersects in two points with $\{a, b, c\},\{a, b, d\}$ and $\{c, d\}$, it follows that $|M \cap B| \geqslant 4$ for some $B \in \bigcup_{i=3}^{5} \mathcal{B}_{i}, B \supset T$. The same arguments apply to the case in which $M \cap\{a, b, c, d\}=\{b, c, d\}$.

If $M \cap\{a, b, c, d\}=\{a, b, c, d\}$ then $M$ is clearly covered by some block $B \in \bigcup_{i=3}^{4} \mathcal{B}_{i}$. The pair $\left(X \cup\{b, c, d\}, \bigcup_{i=1}^{5} \mathcal{B}_{i}\right)$ is therefore a $(v+3,6,4,6)$ cover.

This construction implies the following upper bound formula:

$$
\begin{aligned}
C(v+3,6,4,6) \leqslant & 3\left\lfloor\frac{6}{v} C(v, 6,4,6)\right\rfloor+C(v, 6,4,6)+2 C\left(v_{3,1}+v_{3,2}, 3,2\right) \\
& +C\left(v_{1,1}+v_{2,1}, 3,2\right)+C\left(v_{1,2}+v_{2,2}, 3,2\right) \\
& +C\left(v_{1,1}+v_{2,2}, 3,2\right)+C\left(v_{1,2}+v_{2,1}, 3,2\right) \\
& +C\left(v_{1,1}+v_{1,2}, 4,2\right)+C\left(v_{2,1}+v_{2,2}, 4,2\right)+C\left(v_{3,1}+v_{3,2}, 4,2\right),
\end{aligned}
$$

where $\sum_{i=1}^{3} \sum_{j=1}^{2} v_{i, j}=v-1$.

\section{Trapping-triples Construction for $(v, 6,3, m)$ Covers}

In his paper [12], de Caen presented a construction for $(v, 3, m)$ Turán systems. It was based on the partition of a set $X$ into $m-1$ quasi-equal parts, that is, parts whose sizes pairwise differ by one unit at most. For $m=4$, de Caen's construction coincides with the one given by Turán in [31] who conjectured that it always produces optimal Turán systems with $T(v, 3,4)$ blocks. The conjecture has been shown to be true for $v \leqslant 13([29])$. Etzion et al. [15] extended de Caen's construction to $(v, 4,3, m)$ covers. It can be further extended to $(v, 6,3, m)$ covers as follows: Let $X$ be a set of $v$ elements and $X_{0}, X_{1}, \ldots, X_{m-2}$ be a partition of $X$ into $m-1$ quasi-equal parts. For $i=0,1, \ldots, m-2$, let $\left(X_{i}, \mathcal{B}_{i}\right)$ be a $\left(v_{i}, 2,1\right)$ covering design with $w_{i}$ blocks $B_{i}^{1}, B_{i}^{2}, \ldots, B_{i}^{w_{i}}$, where $w_{i}=\left\lceil v_{i} / 2\right\rceil$, and let us select $h_{i}\left(v_{i}, 4,2\right)$ covering designs $\left(X_{i}, \mathcal{A}_{i}^{1}\right),\left(X_{i}, \mathcal{A}_{i}^{2}\right), \ldots,\left(X_{i}, \mathcal{A}_{i}^{h_{i}}\right)$ such that $\left(X_{i}, \bigcup_{j=1}^{h_{i}} \mathcal{A}_{i}^{j}\right)$ is a $\left(v_{i}, 4,3\right)$ covering design, where $h_{i}=w_{(i+1) \bmod (m-1)}$. Let us define

$$
\mathcal{C}_{i}=\bigcup_{j=1}^{h_{i}} \mathcal{A}_{i}^{j}\left\{B_{(i+1) \bmod (m-1)}^{j}\right\}
$$

where $i=0,1, \ldots, m-2$.

Theorem 14. $\left(X, \bigcup_{i=0}^{m-2} \mathcal{C}_{i}\right)$ is a $(v, 6,3, m)$ cover. 
Proof. Let us analyze how a given $m$-subset $M$ of $X_{0} \cup X_{1} \cup \ldots \cup X_{m-2}$ is covered in three points by some block $C$ in $\mathcal{C}_{0} \cup \mathcal{C}_{1} \cup \ldots \cup \mathcal{C}_{m-2}$ :

1. Let $\left|M \cap X_{l}\right| \geqslant 3$ for some $l \in\{0,1, \ldots, m-2\}$. From the definition of $\bigcup_{j=1}^{h_{l}} \mathcal{A}_{l}^{j}$, it follows that for some $p \in\left\{1,2, \ldots, h_{l}\right\}$ there exists a block $A \in \mathcal{A}_{l}^{p}$ such that $|M \cap A| \geqslant 3$. This implies $|M \cap C| \geqslant 3$ where $C=A \cup B_{(l+1) \bmod (m-1)}^{p}$.

2. Let $\left|M \cap X_{i}\right| \leqslant 2$, for $0 \leqslant i \leqslant m-2$. Then there exists at least one and at most $\left\lfloor\frac{m}{2}\right\rfloor$ different parts $X_{j_{n}}$ of $X$ such that $\left|M \cap X_{j_{n}}\right|=2$. This implies that there must exist $l \in\{0,1, \ldots, m-2\}$ such that $\left|M \cap X_{l}\right|=2$ and $\left|M \cap X_{(l+1) \bmod (m-1)}\right| \geqslant 1$. From the definition of $h_{l}$ and $\mathcal{B}_{(l+1) \bmod (m-1)}$, it follows that there exists $p \in\left\{1,2, \ldots, h_{l}\right\}$ such that $\left|M \cap B_{(l+1) \bmod (m-1)}^{p}\right| \geqslant 1$. Since $\left(X_{l}, \mathcal{A}_{l}^{p}\right)$ is a $\left(v_{l}, 4,2\right)$ covering design, there must exist a block $A \in \mathcal{A}_{l}^{p}$ such that $|M \cap A|=2$ and therefore $|M \cap C| \geqslant 3$ where $C=A \cup B_{(l+1) \bmod (m-1)}^{p}$.

We have therefore shown that, for any $m$-subset $M$ of $X$, there exists a block $C \in \bigcup_{i=0}^{m-2} \mathcal{C}_{i}$ such that $|M \cap C| \geqslant 3$. That is, $\left(X, \bigcup_{i=0}^{m-2} \mathcal{C}_{i}\right)$ is a $(v, 6,3, m)$ cover.

From Theorem 5 and Theorem 6 we can derive a general upper bound formula for $(v, 6,3, m)$ covers.

Theorem 15. Let $n$ be any positive integer. For $v=4^{n}$ or $v=2 p^{n}+2$ with $p \in$ $\{7,31,127\}$, the following inequality holds:

$$
C((m-1) v, 6,3, m) \leqslant \frac{v^{2}(v-1)(m-1)}{24} .
$$

Proof. Let $X$ be a set of $(m-1) v$ points. Let $v=4^{n}$ or $v=2 p^{n}+2$ where $n$ is a positive integer and $p \in\{7,31,127\}$. For $i=0,1, \ldots, m-2$ :

Let $X_{i}$ be a part of $X$ and $\left|X_{i}\right|=v$. Let $B_{i}^{1}, B_{i}^{2}, \ldots, B_{i}^{w}$ be a partition of $X_{i}$ where $\left|B_{i}^{1}\right|=\left|B_{i}^{2}\right|=\ldots=\left|B_{i}^{w}\right|=2$. Therefore $w=\frac{v}{2}$.

From Theorem 5 and Theorem 6 it follows that there exists a Steiner quadruple system $\left(X_{i}, \mathcal{A}_{i}\right)$ which is 2-resolvable. This implies that the collection $\mathcal{A}_{i}$ of blocks can be partitioned into $r$ parts $\mathcal{A}_{i}^{1}, \mathcal{A}_{i}^{2}, \ldots, \mathcal{A}_{i}^{r}$, each of which is the collection of blocks of a Steiner system $S(v, 4,2)$. The value of $r$ is

$$
\frac{v(v-1)(v-2)}{4 \cdot 3 \cdot 2} \cdot \frac{4 \cdot 3}{v(v-1)}=\frac{v-2}{2},
$$

where $\frac{v(v-1)(v-2)}{4 \cdot 3 \cdot 2}$ is the number of blocks of an $\operatorname{SQS}(v)$ and $\frac{v(v-1)}{4 \cdot 3}$ is the number of blocks of an $S(v, 4,2)$. Let $\mathcal{A}_{i}^{h}$ be an additional collection of blocks such that $\mathcal{A}_{i}^{h}=\mathcal{A}_{i}^{r}$ and $h=r+1$. Clearly, $h=\frac{v}{2}=w$. 
We now have all the elements to apply the construction presented in Theorem 14 which develops as follows:

$$
\begin{aligned}
\left|\bigcup_{i=0}^{m-2} \mathcal{C}_{i}\right| & =\left|\bigcup_{j=1}^{h} \mathcal{A}_{0}^{j}\left\{B_{1}^{j}\right\} \cup \bigcup_{j=1}^{h} \mathcal{A}_{1}^{j}\left\{B_{2}^{j}\right\} \cup \ldots \cup \bigcup_{j=1}^{h} \mathcal{A}_{m-2}^{j}\left\{B_{0}^{j}\right\}\right| \\
& =(m-1)\left(\frac{v(v-1)(v-2)}{4 \cdot 3 \cdot 2}+\frac{v(v-1)}{4 \cdot 3}\right) \\
& =(m-1)\left(\frac{v(v-1)(v-2)+2 v(v-1)}{24}\right) \\
& =(m-1)\left(\frac{v(v-1)(v-2+2)}{24}\right) \\
& =\frac{v^{2}(v-1)(m-1)}{24} .
\end{aligned}
$$

As a consequence of Theorem 15, the following upper bound on the minimum size of $(3 v, 6,3,4)$ covers can be stated:

Corollary 16. Let $n$ be any positive integer. For $v=4^{n}$ or $v=2 p^{n}+2$ with $p \in$ $\{7,31,127\}$,

$$
C(3 v, 6,3,4) \leqslant \frac{v^{2}(v-1)}{8}
$$

\section{Trapping-quadruples Constructions}

In the following section we present a construction for $(v, k, 4,6)$ covers and sufficient conditions for its application will be discussed. Then, by requiring additional conditions to be satisfied, a construction for $(v, k, 4,5)$ covers will be derived.

\subsection{Construction of $(v, k, 4,6)$ Covers}

Let $X$ be a set of $v$ elements, $v$ even, and $X_{1}, X_{2}$ be a partition of $X$ into two equal parts. Let $n=\frac{v}{2}$. Moreover, let $k$ be an even number, $k \geqslant 4$ and $h=\frac{k}{2}$. Suppose there exists a resolvable $(n, h, 2)$ covering design with $p$ parallel classes, $p \leqslant 5$. Let $\mathcal{P}_{1}, \mathcal{P}_{2}, \ldots, \mathcal{P}_{p}$ be the parallel classes defined on $X_{1}$ and $\mathcal{R}_{1}, \mathcal{R}_{2}, \ldots, \mathcal{R}_{p}$ be the parallel classes defined on $X_{2}$. For $i=1,2$, let $\left(X_{i}, \mathcal{B}_{i}\right)$ be a $(n, k, 4)$ covering design. We assume therefore that $n \geqslant k>h$. Under this assumption, Theorem 9 implies $p \geqslant 3$. Define

$$
\mathcal{B}=\mathcal{B}_{1} \cup \mathcal{B}_{2} \cup \bigcup_{i=1}^{p} \mathcal{P}_{i} \mathcal{R}_{i} .
$$

Theorem 17. $(X, \mathcal{B})$ is a $(v, k, 4,6)$ cover.

Proof. Let us analyze how a 6 -subset $M$ of $X_{1} \cup X_{2}$ is covered in 4 points by some block $B \in \mathcal{B}$ : 
1. $M \in[6,0] \cup[5,1] \cup[4,2]$. Then there exists a block $B \in \mathcal{B}_{1}$ such that $|B \cap M| \geqslant 4$ since $\left(X_{1}, \mathcal{B}_{1}\right)$ is a $(n, k, 4)$ covering design.

2. $M \in[0,6] \cup[1,5] \cup[2,4]$. Then there exists a block $B \in \mathcal{B}_{2}$ such that $|B \cap M| \geqslant 4$ since $\left(X_{2}, \mathcal{B}_{2}\right)$ is a $(n, k, 4)$ covering design too.

3. $M \in[3,3]$. Let $T=M \cap X_{1}$ and $S=M \cap X_{2}$.

(a) Suppose that the triple $T$ is contained in a block $P$ of a parallel class $\mathcal{P}_{i}$, $i \in\{1,2, \ldots, p\}$. Then from the definition of $\mathcal{R}_{i}$ it follows that there exists a block $R \in \mathcal{R}_{i}$ such that $|R \cap M| \geqslant 1$. This implies $|M \cap B| \geqslant 4$ where $B=P \cup R$. We can proceed symmetrically when the triple $S$ is contained in a block of a parallel class $\mathcal{R}_{j}, j \in\{1,2, \ldots, p\}$.

(b) Suppose instead that $T$ is not contained in any block of any class $\mathcal{P}_{i}, 1 \leqslant i \leqslant p$, and $S$ is not contained in any block of any class $\mathcal{R}_{j}, 1 \leqslant j \leqslant p$. Then for some $i_{1}, i_{2}, i_{3} \in\{1,2, \ldots, p\}$, where $i_{1}<i_{2}<i_{3}$, and for some $j_{1}, j_{2}, j_{3} \in$ $\{1,2, \ldots, p\}$, where $j_{1}<j_{2}<j_{3}$, there must exist $I_{1} \in \mathcal{P}_{i_{1}}, I_{2} \in \mathcal{P}_{i_{2}}, I_{3} \in \mathcal{P}_{i_{3}}$, $J_{1} \in \mathcal{R}_{j_{1}}, J_{2} \in \mathcal{R}_{j_{2}}$ and $J_{3} \in \mathcal{R}_{j_{3}}$ such that $\left|T \cap I_{l}\right|=\left|S \cap J_{l}\right|=2$, for $1 \leqslant l \leqslant 3$. This is because the pairs in $T$ (and the pairs in $S$ ) pairwise intersect in one point and cannot be contained in different blocks of a same parallel class by definition. Since $p \leqslant 5$, there must exist $y, z \in\{1,2,3\}$ such that $i_{y}=j_{z}$. This implies $\left|\left(I_{y} \cup J_{z}\right) \cap M\right|=4$.

We have shown that, for any 6 -subset $M$ of $X$, there exists a block $B \in \mathcal{B}$ such that $|M \cap B| \geqslant 4$. Hence $(X, \mathcal{B})$ is a $(v, k, 4,6)$ cover.

Under the conditions of the construction presented in this section, we have

$$
C(v, k, 4,6) \leqslant 2 C(v / 2, k, 4)+\frac{p v^{2}}{k^{2}} .
$$

Remark 18. From the construction mentioned above, we deduce that it is not always true that a given 6-subset $M$ of $X_{1} \cup X_{2}, M \in[4,2] \cup[2,4]$, is covered in four points by some block $B \in \bigcup_{i=1}^{p} \mathcal{P}_{i} \mathcal{R}_{i}$, but it is true if the size of each parallel class is less than four. Let us investigate the reason. Let $M \in[4,2]$ (the case when $M \in[2,4]$ can be dealt with in a similar way) and suppose that the size of each parallel class is $q<4$. For $i=1,2, \ldots, p$, the four points of the quadruple $M \cap X_{1}$ cannot lie in four different blocks of $\mathcal{P}_{i}$ (as the size of each class is less than four) and therefore $\left|\left(M \cap X_{1}\right) \cap P\right| \geqslant 2$ for some block $P \in \mathcal{P}_{i}$. On the other hand, there exists a parallel class $\mathcal{R}_{j}$, for some $j \in\{1,2, \ldots, p\}$, which contains a block $R$ such that $|M \cap R|=2$. This implies that, for some $P \in \mathcal{P}_{j}$, we have $|M \cap(P \cup R)| \geqslant 4$ and the above-mentioned construction can be improved by replacing $\mathcal{B}_{1}$ and $\mathcal{B}_{2}$ with the collections $\mathcal{C}_{1}$ and $\mathcal{C}_{2}$ of two $(n, k, 4,5)$ covers $\left(X_{1}, \mathcal{C}_{1}\right)$ and $\left(X_{2}, \mathcal{C}_{2}\right)$. This improvement implies the following better upper bound for $(v, k, 4,6)$ covers:

$$
C(v, k, 4,6) \leqslant 2 C(v / 2, k, 4,5)+\frac{p v^{2}}{k^{2}} .
$$




\subsection{Construction of $(v, k, 4,5)$ Covers}

Let us consider again the construction presented in Section 5.1 but instead of requiring that the number of parallel classes be $p \leqslant 5$, we require that the size of the parallel classes be $q=2$.

Theorem 19. $(X, \mathcal{B})$ is a $(v, k, 4,5)$ cover.

Proof. Let us analyze how a 5-subset $M$ of $X_{1} \cup X_{2}$ is covered in 4 points by some block $B \in \mathcal{B}:$

1. $M \in[5,0] \cup[4,1] \cup[1,4] \cup[0,5]$. Then $M$ is covered by some block $B \in \mathcal{B}_{1} \cup \mathcal{B}_{2}$ for the same considerations made in Theorem 17, points 1 and 2.

2. $M \in[3,2] \cup[2,3]$. Let $\left|M \cap X_{1}\right|=3$ (the case when $\left|M \cap X_{2}\right|=3$ can be dealt with in a similar way). For $i=1,2, \ldots, p$, the three points of the triple $M \cap X_{1}$ cannot lie in three different blocks of $\mathcal{P}_{i}$ (as the size of each class is less than three). By similarly following the same arguments made in Remark 18, we deduce that for some $j \in\{1,2, \ldots, p\}$ there exist $P \in \mathcal{P}_{j}$ and $R \in \mathcal{R}_{j}$ such that $|M \cap(P \cup R)| \geqslant 4$.

Under the conditions of the construction presented in Theorem 19, we have

$$
C(v, k, 4,5) \leqslant 2 C(v / 2, k, 4)+4 p \text {. }
$$

Now, let us note that $C(k, k, 4)$ is trivially equal to 1 and that $C(3 k, 2 k, 4,5)=3[9]$. Moreover, since $C(v m, k m, t) \leqslant C(v, k, t)[19]$, we have $C(16 m, 8 m, 4) \leqslant C(16,8,4)=30$ [18]. These facts, combined with Theorem 10, Theorem 17, Remark 18 and Theorem 19, lead to the following upper bounds for covers:

Theorem 20. For $k \geqslant 2$, we have:

1. $C(4 k, 2 k, 4,5) \leqslant 14$ if $k$ is even;

2. $C(4 k, 2 k, 4,5) \leqslant 18$ if $k$ is odd;

3. $C(6 k, 2 k, 4,6) \leqslant 42$ if $k \equiv 0(\bmod 3)$;

4. $C(6 k, 2 k, 4,6) \leqslant 51$ if $k \equiv 1,2(\bmod 3)$;

5. $C(8 k, 2 k, 4,6) \leqslant 140$ if $k \equiv 0(\bmod 4)$.

Points 1 and 2 of Theorem 20 derive from Theorem 10 and Theorem 19. Points 3 and 4 from Theorem 10, Theorem 17 and Remark 18. Point 5 from Theorem 10 and Theorem 17 .

Here below some examples follow, where $p$ indicates the number of parallel classes and $q$ the size of each of them: 
$k=3, p=4$ and $q=3$. In this case, the resolvable $(9,3,2)$ covering design with 12 blocks from Theorem 10 is the well-known resolvable Steiner system $S(9,3,2)$. From Theorem 20 point 3 , we have $C(18,6,4,6) \leqslant 42$, which matches the current best known upper bound for $C(18,6,4,6)^{1}[21]$.

$k=2, p=5$ and $q=3$. In this case, from Theorem 10, we have the 1 -factors of the 1-factorization of the complete graph $K_{6}$. From Theorem 20 point 4, we have $C(12,4,4,6) \leqslant 51$, which matches the best upper bound for $C(12,4,4,6)$ [18], (i.e. for the Turán number $T(12,4,6)$ and therefore for the covering number $C(12,8,6)$ as well).

$k=2, p=3$ and $q=2$. In this case, from Theorem 10, we have the 1 -factors of the 1-factorization of the complete graph $K_{4}$. From Theorem 20 point 1, we obtain $C(8,4,4,5) \leqslant 14$. Since an SQS(8) exists, we have $C(8,4,3)=T(8,4,5)=$ $C(8,4,4,5)=14$. It is worth noting that in this case, from the construction yielding Theorem 19, blocks and coblocks not only have the same size but are identical: the constructed $(8,4,5)$ Turán system and its complement, a Steiner system $S(8,4,3)$, are the same design.

\section{Trapping-quintuples Constructions}

Tables of upper bounds for covers are published in $[5,16,21,22]$. We target values with parameters $k=6, t=5$ and $m=7$ and we provide new upper bounds for $C(v, 6,5,7)$, $(v=26,28,29)$.

Theorem 21. $C(28,6,5,7) \leqslant 2910$.

Proof. Let $X$ be a set such that $|X|=28$. Divide $X$ into two separate sets $X_{1}$ and $X_{2}$ such that $\left|X_{1}\right|=10$ and $\left|X_{2}\right|=18$. Let $X_{2,1}, X_{2,2}$ and $X_{2,3}$ be a partition of $X_{2}$ into three equal parts. For $i=1,2,3$ and $j=1,2, \ldots, 5$, let $\mathcal{B}_{i, j}$ be the 1 -factors of the 1 -factorization of the complete graph $K_{6}$ on $X_{2, i}$. From Theorem 11, there exist ten Steiner quadruple systems $\left(X_{1}, \mathcal{Q}_{1}\right),\left(X_{1}, \mathcal{Q}_{2}\right), \ldots,\left(X_{1}, \mathcal{Q}_{10}\right)$ such that $\bigcup_{i=1}^{10} \mathcal{Q}_{i}=\left(\begin{array}{c}X_{1} \\ 4\end{array}\right)$. Let $\left(X_{1}, \mathcal{D}\right)$ be a $(10,6,5,6)$ cover and $\left(X_{2}, \mathcal{E}\right)$ be a $(18,6,5)$ covering design. Define $\mathcal{A}_{1,1}=\mathcal{Q}_{1}, \mathcal{A}_{1,2}=\mathcal{Q}_{2}$, $\mathcal{A}_{1,3}=\mathcal{Q}_{3}, \mathcal{A}_{1,4}=\mathcal{A}_{1,5}=\mathcal{Q}_{4}, \mathcal{A}_{2,1}=\mathcal{Q}_{5}, \mathcal{A}_{2,2}=\mathcal{Q}_{6}, \mathcal{A}_{2,3}=\mathcal{Q}_{7}, \mathcal{A}_{2,4}=\mathcal{A}_{2,5}=\mathcal{Q}_{4}$, $\mathcal{A}_{3,1}=\mathcal{Q}_{8}, \mathcal{A}_{3,2}=\mathcal{Q}_{9}, \mathcal{A}_{3,3}=\mathcal{Q}_{10}, \mathcal{A}_{3,4}=\mathcal{A}_{3,5}=\mathcal{Q}_{4}$. We claim that

$$
\mathcal{C}=\mathcal{D} \cup \mathcal{E} \cup \bigcup_{i=1}^{3} \bigcup_{j=1}^{5} \mathcal{A}_{i, j} \mathcal{B}_{i, j}
$$

is the collection of blocks of a $(28,6,5,7)$ cover.

Let us analyze how a 7 -subset $M$ of $X_{1} \cup X_{2}$ is covered in 5 points by a block $C \in \mathcal{C}$ :

\footnotetext{
${ }^{1}$ Bertolo et al. (cf. [6]) applied an analogous technique to the case when $v=19, t=4$ and $k=m=6$ from which the bound $C(18,6,4,6) \leqslant 42$ can be derived almost straightforwardly. However, they did not investigate the conditions under which the technique generalizes to other values of $v, k$ and $m$, nor did they determine upper bounds of the kind presented in Theorem 20.
} 
1. $M \in[7,0] \cup[6,1]$. Then $M$ is covered by some block $C \in \mathcal{D}$.

2. $M \in[2,5] \cup[1,6] \cup[0,7]$. Then $M$ is covered by some block $C \in \mathcal{E}$.

3. $M \in[4,3]$ and $\left|M \cap X_{2, i}\right| \geqslant 2$ for some $i \in\{1,2,3\}$. Then, for some $j \in\{1,2,3,4,5\}$, there exists a block $B$ belonging to $\mathcal{B}_{i, j}$ such that $|M \cap B|=2$. Since $\left(X_{1}, \mathcal{A}_{i, j}\right)$ is a $(10,4,3)$ covering design, there must exist a block $A \in \mathcal{A}_{i, j}$ such that $|M \cap A| \geqslant 3$ and therefore $|M \cap C| \geqslant 5$ where $C=A \cup B$.

4. $M \in[4,3]$ and $\left|M \cap X_{2, i}\right|=1$ for $i=1,2,3$. Since $\bigcup_{i=1}^{3} \bigcup_{j=1}^{5} \mathcal{A}_{i, j}$ is the collection of all the quadruples from $X_{1}$, for some $r \in\{1,2,3\}$ and some $s \in\{1,2,3,4,5\}$ there exists a block $A \in \mathcal{A}_{r, s}$ such that $|M \cap A|=4$. On the other hand, there exists a block $B \in \mathcal{B}_{r, s}$ such that $|M \cap B|=1$. This implies $|M \cap C|=5$ where $C=A \cup B$.

5. $M \in[3,4]$. In this case, at least two points of $M \cap X_{2}$ belong to $X_{2, i}$ for some $i \in\{1,2,3\}$. Therefore $\left|M \cap X_{2, i}\right| \geqslant 2$ and the same arguments made at point 3 apply.

6. $M \in[5,2]$ and $\left|M \cap X_{2, i}\right|=2$ for some $i \in\{1,2,3\}$. Again, the same arguments made at point 3 apply.

7. $M \in[5,2]$ and $\left|M \cap X_{2, p_{1}}\right|=1,\left|M \cap X_{2, p_{2}}\right|=1$ for some $p_{1}, p_{2} \in\{1,2,3\}, p_{1}<p_{2}$. Note that the five quadruples from $M \cap X_{1}$ belong to the collections of blocks of five different Steiner quadruple systems $\left(X_{1}, \mathcal{Q}_{i_{1}}\right),\left(X_{1}, \mathcal{Q}_{i_{2}}\right), \ldots,\left(X_{1}, \mathcal{Q}_{i_{5}}\right)$, where $i_{1}, i_{2}, \ldots, i_{5} \in\{1,2, \ldots, 10\}$ and $i_{1}<i_{2}<\ldots<i_{5}$. This is because they pairwise intersect in three points and two quadruples cannot intersect in three points if they belong to the same Steiner quadruple system. From the definition of $\bigcup_{i=1}^{3} \bigcup_{j=1}^{5} \mathcal{A}_{i, j}$, it follows that for some $r_{1}, r_{2} \in\{1,2,3\}$, where $r_{1}<r_{2}$, and for some $s_{1}, s_{2} \in$ $\{1,2,3,4,5\}$, there exist two blocks $A_{1} \in \mathcal{A}_{r_{1}, s_{1}}$ and $A_{2} \in \mathcal{A}_{r_{2}, s_{2}}$ such that $\left|M \cap A_{1}\right|=$ 4 and $\left|M \cap A_{2}\right|=4$. It must also be the case that $\left\{r_{l}\right\} \cap\left\{p_{1}, p_{2}\right\} \neq \emptyset$ for some $l \in\{1,2\}$ as $X_{2}$ is split into three parts. For this $l$, there exists a block $B \in \mathcal{B}_{r_{l}, s_{l}}$ such that $|M \cap B|=1$ and therefore $|M \cap C|=5$ where $C=A_{l} \cup B$.

We have shown that, for any 7-subset $M$ of $X$, there exists a block $C \in \mathcal{C}$ such that $|M \cap C| \geqslant 5$. Since $C(10,6,5,6) \leqslant 14, C(10,4,3)=30$ and $C(18,6,5) \leqslant 1546([16,18])$, the following inequality holds:

$$
\begin{aligned}
C(28,6,5,7) & \leqslant C(10,6,5,6)+C(18,6,5)+45 C(10,4,3) \\
& \leqslant 14+1546+1350 \\
& =2910 .
\end{aligned}
$$

The previous best-known bound was 2952 ([16]).

The upper bound $C(26,6,5,7) \leqslant 1872$ (old best-known bound 1897 [16]) can be obtained similarly by splitting the starting set $X$ into four parts $X_{1}, X_{2,1}, X_{2,2}$ and $X_{2,3}$ such that $\left|X_{1}\right|=10,\left|X_{2,1}\right|=6,\left|X_{2,2}\right|=\left|X_{2,3}\right|=5$. 
Theorem 22. $C(29,6,5,7) \leqslant 3627$.

Proof. Let $X=\{a, 2,3, \ldots, 28\}, X_{1}=\{a, 2,3, \ldots, 10\}$ and $X_{2}=\{11,12, \ldots, 28\}$. Let $(X, \mathcal{C})$ be a $(28,6,5,7)$ cover obtained by applying the construction of Theorem 21 . From the same construction, let $\left(X_{1}, \mathcal{D}\right)$ be the following $(10,6,5,6)$ cover computed by means of the software described in [27]:

$$
\begin{aligned}
\left(X_{1}, \mathcal{D}\right)=\left(X_{1},\right. & \{\{a, 2,3,4,5,6\},\{a, 2,3,6,7,9\},\{a, 2,4,7,9,10\},\{a, 2,4,8,9,10\}, \\
& \{a, 3,4,6,8,10\},\{a, 3,5,7,9,10\},\{a, 4,5,7,8,9\},\{a, 5,6,7,8,10\}, \\
& \{2,3,4,6,7,8\},\{2,3,5,6,8,9\},\{2,3,5,7,8,10\},\{2,4,5,6,9,10\}, \\
& \{3,4,5,7,9,10\},\{4,6,7,8,9,10\}\}) .
\end{aligned}
$$

We will show that the element $a$ occurs 548 times in $\mathcal{C}$. By applying the point splicing construction of Section 3.1, we will have:

$$
C(29,6,5,7) \leqslant 548+|\mathcal{C}|+C(27,4,3,5) \text {. }
$$

Given a set $V$, if there exists a Steiner quadruple system $(V, \mathcal{A})$, every element $v \in V$ occurs exactly $\frac{4|\mathcal{A}|}{v}$ times in $\mathcal{A}$. In our case, since $\left|\bigcup_{i=1}^{3} \bigcup_{j=1}^{5} \mathcal{B}_{i, j}\right|=45$, the element $a$ occurs $45 \cdot \frac{4 \cdot 30}{10}=540$ times in $\bigcup_{i=1}^{3} \bigcup_{j=1}^{5} \mathcal{A}_{i, j} \mathcal{B}_{i, j}$. Moreover, a occurs 8 times in $\mathcal{D}$ and therefore 548 times in $\mathcal{C}$. Since $C(27,4,3,5) \leqslant C(13,4,3)+C(14,4,3)=78+91=169$ (see $[18,21]$ ) and, from Theorem $21,|\mathcal{C}|=2910$, we have $C(29,6,5,7) \leqslant 3627$.

Note: The 3627 blocks of the cover presented in Theorem 22 have been transformed and further reduced to 3607 blocks by local search. The blocks are listed in [26].

\section{Conclusions}

Improving upper bounds on the minimum size of general covering designs is a challenging problem. In order to obtain good upper bounds, combinatorial constructions involving unions and intersections of different kind of combinatorial designs are very effective and we think it is worth keeping exploiting these techniques.

\section{Acknowledgements}

The author would like to thank Prof. Peter J. Cameron for his helpful comments and an anonymous referee whose suggestions and remarks helped improve the presentation of this paper.

\section{References}

[1] R.F. Bailey, A.C. Burgess, M.S. Cavers, K. Meagher. Generalized covering designs and clique coverings, J. Combin. Des. 19 (2011), 378-406. 
[2] R.D. Baker. Partitioning the planes of $A G_{2 m}(2)$ into 2-designs, Discrete Math. 15 (1976), 205-211.

[3] J.A. Bate, R.G. Stanton. Some algorithmic results on $(2,3,3, v)$ designs, $(2,4,4, v)$ designs, Util. Math. 20 (1981), 195-220.

[4] J.A. Bate, G.H.J. van Rees. Lotto designs, Util. Math. 20 (1990), 195-220.

[5] R. Belic. Complete list of lotto systems, http://rbelic.home.xs4all.nl/lista. htm (accessed June 10, 2011).

[6] R. Bertolo, I. Bluskov, H. Hämäläinen. Upper bounds on the general covering number $C_{\lambda}(v, k, t, m), J$. Combin. Des. 12 (2004), 362-380.

[7] N. Bougard. The lotto numbers L(n,3,p,2), J. Combin. Des. 14 (2006), 333-350.

[8] A.E. Brouwer, M. Voorhoeve. Turán theory and the lotto problem, Math. Centrum Tracts 106 (1979), 99-105.

[9] A.P. Burger, W.R. Gründlingh, J.H. van Vuuren. Towards a characterisation of lottery set overlapping structures, Ars Combin. 84 (2007), 105-128.

[10] A.H. Chan, R.A. Games. $(n, k, t)$-covering systems and error-trapping decoding, IEEE Trans. Inform. Theory 27 (1981), 643-646.

[11] P. Crescenzi, F. Montecalvo, G. Rossi. Optimal covering designs: complexity results and new bounds, Discrete Appl. Math. 144 (2004), 281-290.

[12] D. de Caen. A note on the probabilistic approach to Turán's problem, J. Combin. Theory, Ser. B 34 (1983), 340-349.

[13] D. de Caen, D.L. Kreher, J. Wiseman. On constructive upper bounds for the Turán numbers, Congr. Numer. 65 (1988), 277-280.

[14] T. Etzion. Large sets of coverings, J. Combin. Des. 2 (1994), 359-374.

[15] T. Etzion, V. Wei, Z. Zhang. Bounds on the sizes of constant weight covering codes, Des. Codes Cryptogr. 5 (1995), 217-239.

[16] W. Fromme. Fromme's lotto systems site, http://www.weefs-lottosysteme.de (accessed July 27, 2012).

[17] Z. Füredi, G.J. Székely, Z. Zubor. On the lottery problem, J. Combin. Des. 4 (1996) $5-10$.

[18] D. Gordon. La Jolla covering repository, http://www . ccrwest. org/cover .htm (accessed July 27, 2012).

[19] D.M. Gordon, G. Kuperberg, O. Patashnik. New constructions for covering designs, J. Combin. Des. 3 (1995), 269-284.

[20] T.P. Kirkman. On a problem in combinations, Cambridge and Dublin Math. J. 2 (1847), 191-204.

[21] P.C. Li, G.H.J. van Rees. Lotto designs, in The CRC Handbook of Combinatorial Designs, C. J. Colbourn and J. H. Dinitz (Editors), CRC press, 2007, pp. 512-519.

[22] P.C. Li, G.H.J. van Rees. Lotto designs tables, J. Combin. Des. 10 (2002), 335-359. 
[23] P.C. Li, G.H.J. van Rees. Lower bounds on lotto designs, Congr. Numer. 141 (1999), $5-30$.

[24] W.H. Mills. Constructions of covering designs, Congr. Numer. 73 (1990), 29-36.

[25] W.H. Mills, R.C. Mullin. Coverings and packings, in Contemporary Design Theory: A collection of surveys (eds. J.H. Dinitz and D.R. Stinson), Wiley, 1992, 371-399.

[26] F. Montecalvo. (2012, July 5). A (29,6,5,7) lotto design with 3607 blocks, https: //docs . google.com/open?id=0BzDGzgSTFaawdXhvRVA10Uo2b1k.

[27] K.J. Nurmela, P.R.J. Östergård. Upper bounds for covering designs by simulated annealing, Congr. Numer. 96 (1993), 93-111.

[28] A. Sidorenko. What we know and what we do not know about Turán numbers, Graph. Combin. 11 (1995), 179-199.

[29] T.H. Spencer. On the size of independent sets in hypergraphs, in Coding Theory, Design Theory, Group Theory, Proceedings of the Marshall Hall Conference, Vermont, 1990 (D. Jungnickel and S. A. Vanstone, Eds.), Wiley, New York, 1993, pp. 263-273.

[30] L. Teirlinck. Some new 2-resolvable Steiner quadruple systems, Des. Codes Cryptogr. 4 (1994), 5-10.

[31] P. Turán. Research problems, Maguar Tud. Akad. Mat. Kutato Int. Közl. 6 (1961), 417-423.

[32] E.R. van Dam, W.H. Haemers, M.B.M. Peek. Equitable resolvable coverings, J. Combin. Des. 11 (2003), 113-123. 